\title{
Cultural differences in acceptability of a vaginal microbicide: a comparison between potential users from Nashville, Tennessee, USA, and Kafue and Mumbwa, Zambia
}

This article was published in the following Dove Press journal:

HIVIAIDS - Research and Palliative Care

8 May 2012

Number of times this article has been viewed

\author{
Valerie Montgomery Rice' \\ Margaret C Maimbolwa ${ }^{2}$ \\ Esther Munalula Nkandu² \\ Jacqueline Fleming \\ Hampton ${ }^{3, *}$ \\ Jae-Eun Lee ${ }^{4}$ \\ James EK Hildreth ${ }^{5}$
}

'Morehouse School of Medicine, Atlanta, GA, USA; ${ }^{2}$ School of Medicine, University of Zambia, Lusaka,

Zambia; ${ }^{3}$ Meharry Medical College,

Center for AIDS Health Disparities

Research, Nashville, TN, USA; ${ }^{4}$ RCMI

Translational Research Network Data and Technology Coordinating Center, Jackson State University, Jackson, MS, USA; ${ }^{5}$ Dean, College of Biological Sciences, University of California Davis, Davis, CA, USA

Note: at the time the research was conducted, Dr Montgomery Rice was the Executive Director, Center for Women's Health Research, Meharry Medical College, Nashville, TN, USA, and Dr Hildreth was the Director, Center for AIDS Health Disparities Research, Meharry Medical College, Nashville, TN

*Dr Fleming Hampton has passed away since this manuscript was written. We wish to honor her memory by including her as an author

Correspondence: Valerie Montgomery Rice Morehouse School of Medicine,

720 Westview Drive, SW, Hugh Gloster

Building, Suite 100

Atlanta, GA 30310-1495 USA

Tel + | 404752 I728

Fax +| 404752 I594

Email vmrice@msm.edu
Purpose: We sought to determine the relationship between acceptability of a hypothetical vaginal microbicide, cultural factors, and perceived HIV risk among African-American women in Nashville, TN, USA, and African women in Kafue and Mumbwa, Zambia.

Patients and methods: Women in both sites completed a survey. Regression analyses were performed on valid samples (Nashville, 164; Zambia, 101) to determine cultural differences affecting microbicide acceptability. Regression analyses also tested whether individual risk perception affected acceptability.

Results: In Zambia, $89.6 \%$ of women were willing to use a microbicide versus $81.6 \%$ in Nashville $(P<0.0001)$. One cultural difference is that women in the Zambian cohort viewed risk of HIV infection as distinct from risk of acquiring STIs, with $48 \%$ believing they were certain to become infected with AIDS, compared to $4 \%$ of Nashville participants.

Conclusion: These results suggest a high degree of acceptability toward use of a vaginal microbicide to prevent HIV infection.

Keywords: HIV/AIDS, women, birth control, sexually transmitted infections

\section{Introduction}

African-American and African women are disproportionately affected by HIV-1. One potential woman-initiated method to prevent transmission of HIV-1 is use of a vaginal microbicide. ${ }^{1}$ However, without user acceptance, adherence is likely to be low. For this reason, acceptability studies have become key components of microbicide clinical studies. Earlier studies have defined acceptability as "use" and "use preferences." More recently, acceptability trials have related acceptability to HIV risk perception $^{2,3}$ and the effect of cross-cultural experiences. ${ }^{4,5}$

This article presents data from a social science study conducted in Nashville, TN, USA, and Kafue and Mumbwa, Zambia, to determine whether differences exist in levels of acceptability, cultural factors, and/or perceived risk of HIV-1 among potential users of a vaginal microbicide.

\section{Methods}

The microbicide acceptability study was conducted among African-American women living in Nashville, TN, USA, and among African women living in the Kafue and Mumbwa districts in Zambia. Each woman signed a consent form prior to participating. 
In Nashville, TN, USA, women were recruited through the use of email alerts, flyers, and those visiting the Center for Women's Health Research. The women in Zambia were identified through local clinics and local leadership; the criterion was women who had children younger than age 5 years who were being brought to the district hospital for vaccination. Meharry Medical School reviewed and approved the consent forms for the participants in Nashville, TN, USA, as did the University of Zambia for participants from Zambia. Participants completed a 49-question survey that captured their beliefs about use of a vaginal cream, foam, or gel that could be used during sexual intercourse to prevent HIV infection. They could refuse to answer any of the questions and in no case was a participant identified. In Zambia, two researchers administered the questionnaires, as they were familiar with languages used in each of the districts studied. Each woman was asked if she was willing to participate in the study and after meeting the inclusion criteria and consenting, interviews were carried out in a private room. On completion of the interview, the participant was given $\mathrm{K} 70,000$ (US\$15) for transport.

In Nashville, a request to participate in an online survey was sent to a predetermined email list of potential participants who were women aged 18-60 years who represented diverse socioeconomic and ethnic groups. The email list comprised Meharry students, faculty and employees and those women who had provided their email addresses to the Center for Women's Health Research from previous visits to the center or a previous engagement at the Center for an educational or research activity. The survey was accessed via a Web address. The survey questionnaire comprised 65 questions that focused on gaining knowledge from women regarding microbicides, preferences for use, and expectations for effectiveness and partner acceptability. Each woman entered her own responses, which were encrypted and stored in a password-protected database on a Meharry Web server. To maintain anonymity of survey respondents, subjects who took the survey online were not given incentives.

\section{Major outcome measures}

At the USA site, where the survey was completed online, the subjects read the introductory paragraph and then proceeded to answer the questions. At the Zambian site, because most women could not read, an introductory paragraph was read to them followed by each of the questions with the answers recorded, based on the response. Also at the Zambian site, both the product and the applicator were available for the purposes of demonstration. Use of tampons is common in Zambia, even in rural areas like Mumbwa; therefore, the participants were familiar with the concept of a vaginal applicator. Microbicide acceptability was measured by asking the Zambian participants to see, feel, and smell and/or taste the microbicide gel so as to determine their willingness to use the product that would be "placed" in the vagina to prevent HIV infection. At both sites, participants selected one of the following options: "Definitely yes," "Probably," "Probably not," and "Definitely not." A dichotomized variable ("Yes, definitely" versus all others) was used for the categorical data analysis.

Another measure for acceptability was derived by asking participants to rate acceptability with using a microbicide under nine conditions describing its potential features (Table 1). To create a composite measure, we assigned 1 point to "Definitely unacceptable," 2 to "Probably unacceptable,"

Table I Conditional acceptability of microbicide

\begin{tabular}{|c|c|c|c|c|c|c|c|c|}
\hline \multirow[t]{2}{*}{ Condition } & \multicolumn{2}{|c|}{$\begin{array}{l}\text { Total } \\
n=265\end{array}$} & \multicolumn{2}{|c|}{$\begin{array}{l}\text { Nashville } \\
\mathrm{n}=164\end{array}$} & \multicolumn{2}{|c|}{$\begin{array}{l}\text { Zambia } \\
\mathbf{n}=\mid \mathbf{0} \mathbf{I}\end{array}$} & \multicolumn{2}{|c|}{ Chi-square* } \\
\hline & $\mathbf{n}$ & $\%$ & $\mathbf{n}$ & $\%$ & $\mathbf{n}$ & $\%$ & Value & P-value \\
\hline If microbicide were a vaginal suppository & $15 \mid$ & 75.5 & 97 & 78.2 & 54 & 71.1 & 1.31 & 0.2523 \\
\hline If microbicide were a cream & 158 & 77.8 & 91 & 72.2 & 67 & 87.0 & 6.06 & 0.0138 \\
\hline A small amount leaks out of your vagina after sex & 99 & 52.1 & 69 & 57.0 & 30 & 43.5 & 3.23 & 0.0722 \\
\hline $\begin{array}{l}\text { If the microbicide made your vagina wetter than } \\
\text { you normally are during sex }\end{array}$ & 124 & 62.6 & 102 & 83.6 & 22 & 28.9 & 59.77 & $<0.0001$ \\
\hline $\begin{array}{l}\text { If the microbicide made you drier than you normally } \\
\text { are during sex }\end{array}$ & 65 & 33.3 & 27 & 21.8 & 38 & 54.3 & 56.84 & $<0.000$ I \\
\hline If the microbicide has a pleasant taste & 146 & 80.2 & 92 & 80.7 & 54 & 79.4 & 0.04 & 0.8326 \\
\hline $\begin{array}{l}\text { If the microbicide needs to be inserted at least } 15 \text { minutes } \\
\text { before having sex }\end{array}$ & 113 & 58.5 & 76 & 61.8 & 37 & 52.9 & 1.47 & 0.2259 \\
\hline If the microbicide is placed in the vagina with an applicator & 156 & 80.0 & 101 & 90.6 & 55 & 58.3 & 25.69 & $<0.000$ l \\
\hline If the microbicide is placed in the vagina with the fingers & 133 & 70.4 & 86 & 69.9 & 47 & 71.2 & 0.03 & 0.8527 \\
\hline
\end{tabular}

Notes: Number is the frequency and proportion of participants who indicated that the specific condition was probably and definitely acceptable. $*$ Chi-square test for the difference between Nashville and Zambia. 
3 to "Probably acceptable," and 4 to "Definitely acceptable." The conditional acceptability as a composite measure was generated by averaging the scores assigned to each condition. Cronbach's alpha for responses from nine items was 0.79 ( 0.88 for Nashville and 0.70 for Zambia), which was higher than 0.70 as a sign of acceptable internal consistency and reliability. The correlation coefficient between willingnessto-use and composite conditional acceptability was 0.45 $(P<0.0001)$, meaning that cross-structure validity of two acceptability measures was supported. The perceived risk for sexually transmitted infection (STIs) as a key independent variable was assessed by asking participants to indicate the extent of agreement with the 8 statements describing the sexually transmitted infection (Table 3). The participants checked one of the following options: "Strongly agree," "Agree," "Disagree," and "Strongly disagree." We used a dichotomized variable (agree versus disagree) for the analysis.

\section{Statistical methods}

Most statistical analyses were carried out using SAS version 9.1 (SAS Institute Inc, Cary NC). Baseline characteristics and level of acceptability were compared between study sites using a $t$-test for continuous variables and Pearson chisquare tests for categorical variables. To assess the attitude toward the potential features of the microbicide, frequency and percentages were calculated and bar charts used for graphical presentation.

To determine the association of acceptability with background characteristics, perceived risk for acquiring STIs, sexual behaviors, and history of STIs, we conducted a series of logistic regressions for dichotomized acceptability (willingness-to-use) and a series of Analysis of Variance (ANOVA) for continuous acceptability (composite conditional acceptability). Before we conducted the data analyses, we checked the normality of the composite measure of acceptability. It was close to normal distribution ( skewedness $=0.56$ and Kurtosis $=0.36$ ). The results from logtransformed data did not differ from those of nontransformed data. We reported the results from nontransformed data. The regression analyses were separately performed by study sites to determine any cultural difference in factors impacting acceptability. For both logistic regression and ANOVA, we used the Generalized Linear Model (GLM) in which the acceptance was the dependent variable and individual potential factor was the main predictor. The following regression equation was used for the GLM: $g(E[y])=\beta_{0}+\beta_{m} x_{m}$ where $g$ is the link function defining the relationship between the outcome and the linear predictor, which is binomial logit function for logistic regression and identity function for ANOVA; $\beta_{0}$ is a parameter to estimate the intercept and $\beta_{m}$ is a parameter to estimate the contribution of main predictor to the outcome. We reported mean, standard deviation, F-value, and $P$-value for ANOVA and frequency, percent, odds ratio, 95\% confidence interval, and $P$-value for logistic regression.

\section{Results Characteristics of participants}

A total of 101 women from Kafue and Mumbwa districts, Zambia, and 164 from Nashville were included in the study. Major characteristics of participants at each study site are summarized in Table 2. The average age was higher in Nashville (36 years) than in Zambia (31 years). A higher proportion of participants in Nashville were more educated, employed/doing paid work, had medical insurance, and a doctor or other medical provider to see regularly. The majority of the participants from Zambia had low education. The study sites in Zambia were from the rural parts of Zambia which contributed to their low economical and educational status. Further, participants from Zambia were more likely to be married/live with partners than those from Nashville.

\section{Attitude toward use of potential features of microbicide}

If participants replied they and their partners had never used a vaginal method of birth control - eg, cream, foam, gel, vaginal capsule (suppository), or sponge - they were asked to cite reasons for non-use. A total of 249 valid reasons for non-use of the potential microbicide features were identified from 175 participants. "Happy with other methods of birth control" was found to be the most frequent reason for non-use, followed by "never heard of them," "too messy," "not effective in preventing pregnancy," and "not enough information on the product." These five reasons comprised $70 \%$ of the total.

However, this pattern differed between study sites. In Nashville, "being happy with other methods" (39\%) was the most frequent, followed by "too messy" (12\%), "never heard of them" $(10 \%)$, and "not effective in preventing pregnancy $(9 \%)$ and disease (6\%)." In Zambia, two reasons related to lack of information ("never heard of them," 24\%, and "not enough information on the product," 14\%) were the most frequent, followed by "not effective in preventing pregnancy" (9\%), "happy with other methods" (8\%) and "not effective in preventing disease" (7\%). While suspicion about its effectiveness was a greater reason in Nashville, lack of information was 
Table 2 Characteristics of women enrolled in each study site $(n=265)$

\begin{tabular}{|c|c|c|c|c|c|c|}
\hline \multirow[t]{2}{*}{ Characteristics } & \multicolumn{2}{|c|}{ Nashville $(n=164)$} & \multicolumn{2}{|c|}{ Zambia $(n=10 I)$} & \multicolumn{2}{|c|}{ Chi-square } \\
\hline & $\mathbf{n}$ & $\%$ & $\mathbf{n}$ & $\%$ & Value & $P$-value \\
\hline \multicolumn{7}{|l|}{ Marital status } \\
\hline Not married/live alone & 73 & 60.3 & 24 & 24.0 & 29.3446 & $<0.0001$ \\
\hline Married/live with partner & 48 & 39.7 & 76 & 76.0 & & \\
\hline \multicolumn{7}{|l|}{ Years of education } \\
\hline Low education ( $1-12$ years) & 6 & 5.3 & 93 & 93.9 & 178.6293 & $<0.0001$ \\
\hline High education (13+ years) & 108 & 94.7 & 2 & 2.1 & & \\
\hline \multicolumn{7}{|l|}{ Paid work } \\
\hline Unemployed/non-paid work & 28 & 23.0 & 76 & 79.2 & $68.056 \mathrm{I}$ & $<0.0001$ \\
\hline Employed/paid work & 94 & 77.1 & 20 & 20.8 & & \\
\hline Mean age (SD) & 36.4 & 12.6 & 31.1 & 10.4 & & 0.0015 \\
\hline \multicolumn{7}{|l|}{ Any medical insurance } \\
\hline No & 10 & 9.1 & 96 & 96.0 & $158.27 \mid 7$ & $<0.0001$ \\
\hline Yes & 100 & 90.9 & 4 & 4.0 & & \\
\hline \multicolumn{7}{|l|}{ See medical professionals regularly } \\
\hline No & 15 & 12.2 & 78 & 78.0 & 98.2426 & $<0.0001$ \\
\hline Yes & 108 & 87.8 & 22 & 22.0 & & \\
\hline \multicolumn{7}{|c|}{ More than 12 months since last period } \\
\hline No & 86 & 74.1 & 95 & 94.1 & 15.4846 & $<0.0001$ \\
\hline Yes & 30 & 25.9 & 6 & 5.9 & & \\
\hline \multicolumn{7}{|c|}{ Menopausal therapy (if reply to previous question about last period is "yes") } \\
\hline No & 119 & 97.5 & 90 & 96.8 & 0.1144 & 0.7352 \\
\hline Yes & 3 & 2.5 & 3 & 3.2 & & \\
\hline
\end{tabular}

greater in Zambia. The five most frequent reasons comprised $76 \%$ of the total among the Nashville participants, compared with $63 \%$ among those in Zambia, suggesting that more diverse reasons were identified in Zambia than in Nashville.

Specific reasons to like a birth control cream, foam, gel, vaginal capsule (suppository), or sponge for the purposes of preventing HIV acquisition and/or pregnancy among participants from both sites are summarized in Figure 1. A total of 189 valid reasons were identified from 56 participants. "Easy to use" was the most frequent reason to like, followed by "affordable," "no allergic reaction," "convenient," "not need a doctor to get it," and "effective at preventing pregnancy." Among the top 12 reasons, four were convenience-related ("easy to use," "not need a doctor to get it," "not too messy," and "being convenient"); three were related to potential side effects ("non-allergic reaction by user and partner," "no burning," and "wetter vagina") two were financial ("affordable" and "no insurance needed"); and one was related to effectiveness ("preventing pregnancy").

\section{Acceptability of microbicides}

The majority of participants $(85 \%)$ indicated they were willing to consider using a product that could be "placed" in the vagina that could prevent sexually transmitted infections and HIV/AIDS. The acceptance rate was higher in Zambia than in Nashville (89.6\% for Zambia and $81.6 \%$ for Nashville; $P<0.0001)$. Those from Zambia had stronger willingness than those from Nashville. While $81 \%$ of Zambian women indicated that they would "Definitely" consider it, only $53 \%$ participants from Nashville did.

We asked participants to rate their level of acceptability with using a microbicide under the nine conditions describing its potential features. Table 1 shows the frequency and proportion of participants who indicated that the specific condition was "probably" or "definitely" acceptable. The participants rated the highest acceptability for "pleasant taste" and "placement in the vagina with an applicator" and only one in three (33.3\%) indicated that "dryness" was acceptable. This pattern was different between sites. While a higher acceptance was reported on "wetness" and "placement in vagina" among participants in Nashville, participants in Zambia rated higher on "cream" and "dryness." "Wetness" and "placement in vagina" were one of the highest rated conditions in Nashville; however, the Zambian cohort rated this lowest. While the majority (54.3\%) of Zambian women indicated that "dryness" was acceptable, only one in five (21.8\%) of participants in Nashville concurred. Mean scores of composite conditional acceptability were $2.7(\mathrm{SD}=0.7)$. 
Table 3 Results of GLM separated by sites to determine association between conditional acceptability and perceived risk for acquiring STIs

\begin{tabular}{|c|c|c|c|c|c|c|c|c|}
\hline & \multicolumn{4}{|c|}{ Nashville } & \multicolumn{4}{|c|}{ Zambia } \\
\hline & \multicolumn{2}{|c|}{$\begin{array}{l}\text { Conditional } \\
\text { acceptability }\end{array}$} & \multicolumn{2}{|c|}{ ANOVA } & \multicolumn{2}{|c|}{$\begin{array}{l}\text { Conditional } \\
\text { acceptability }\end{array}$} & \multicolumn{2}{|c|}{ ANOVA } \\
\hline & Mean & SD & $\mathbf{F}$ & $P$-value & Mean & SD & $\mathbf{F}$ & P-value \\
\hline \multicolumn{9}{|c|}{ Regular consultations "checks" and wellness exams with a health care provider is the best way for me to avoid a sexually transmitted disease } \\
\hline Disagree & 2.8 & 0.6 & 0.08 & 0.7810 & 2.3 & 0.9 & 3.9 & 0.0515 \\
\hline Agree & 2.8 & 0.6 & & & 2.7 & 0.8 & & \\
\hline \multicolumn{9}{|c|}{ No matter what I do I am likely to get a sexually transmitted infection } \\
\hline Disagree & 2.8 & 0.6 & 0.74 & 0.3902 & 2.5 & 0.9 & 0.9 & 0.3497 \\
\hline Agree & 3.0 & 0.8 & & & 2.7 & 0.9 & & \\
\hline \multicolumn{9}{|c|}{ My partner has a lot to do with my getting or avoiding a sexually transmitted disease } \\
\hline Disagree & 2.8 & 0.6 & 0.00 & 0.9477 & 2.3 & 0.9 & 2.8 & 0.1012 \\
\hline Agree & 2.8 & 0.6 & & & 2.7 & 0.8 & & \\
\hline \multicolumn{9}{|c|}{ Luck plays a big part in determining if I can keep from getting a sexually transmitted infection } \\
\hline Disagree & 2.8 & 0.6 & 5.59 & 0.0197 & 2.6 & 0.7 & 0.0 & 0.9276 \\
\hline Agree & 3.4 & 0.6 & & & 2.6 & 0.9 & & \\
\hline \multicolumn{9}{|c|}{ If I take the right actions, I can avoid getting a sexually transmitted infection } \\
\hline Disagree & 2.9 & 0.2 & 0.04 & 0.8355 & 2.5 & 1.0 & 0.3 & 0.5812 \\
\hline Agree & 2.8 & 0.6 & & & 2.7 & 0.8 & & \\
\hline \multicolumn{9}{|c|}{ I refuse to have sex if my partner refuses to use a condom or latex barrier } \\
\hline Disagree & 2.8 & 0.5 & 0.02 & 0.8889 & 2.3 & 0.8 & 10.0 & 0.0023 \\
\hline Agree & 2.8 & 0.6 & & & 2.9 & 0.8 & & \\
\hline \multicolumn{9}{|c|}{ I feel almost certain that I will get AIDS } \\
\hline Disagree & 2.8 & 0.6 & 4.04 & 0.0467 & 2.3 & 0.8 & 4.5 & 0.0363 \\
\hline Agree & 2.3 & 0.7 & & & 2.7 & 0.9 & & \\
\hline \multicolumn{9}{|c|}{ My past behavior puts me at high risk for getting HIV or AIDS } \\
\hline Disagree & 2.7 & 0.6 & 1.49 & 0.2243 & 2.4 & 0.7 & 16.6 & 0.0001 \\
\hline Agree & 2.9 & 0.5 & & & 3.1 & 0.8 & & \\
\hline
\end{tabular}

Abbreviations: STIs, sexually transmitted infections; GLM, generalized linear model.

The scores were slightly higher among the participants in Nashville than in Zambia (2.8 for Nashville and 2.6 for Zambia; $P=0.0855$ ).

\section{Association of acceptability with major characteristics}

The participants indicated a high willingness-to-use, regardless of subgroups of marital status, years in education, employment status, and availability of medical professionals. However, willingness-to-use differed by the subgroups of age and site. The separated logistic regression analyses suggested that older participants (aged 40+ years) in Nashville were more likely to be willing to use than their younger counterparts aged $18-39$ years $(71.4 \%$ for older and $42.4 \%$ for younger; $P=0.004)$, while younger participants in Zambia were more willing to use than older ones $(58.8 \%$ for older and $86.1 \%$ for younger; $P=0.013$ ).

Most of the characteristic variables were not significantly associated with composite measure of conditional acceptability in either site with only one exception; in Zambia, women who were not married or lived alone reported a higher conditional acceptability than their counterparts $(P=0.036)$.

\section{Association of acceptability with perceived risk for STIs}

A woman's willingness-to-use a vaginal microbicide was not associated with degree of agreement with eight statements describing perceived risk for STIs, with one exception: in Zambia, $87 \%$ of those who agreed "feel almost certain they will get AIDS" and indicated they were willing to use it, while $68 \%$ of those who disagreed indicated that they were willing to do so $(P=0.049)$.

Table 3 shows the results of ANOVA analyses to determine the association between conditional acceptability and perceived risk for acquiring STIs. Women who perceived a high risk for STIs were more likely to accept the potential features of a vaginal microbicide. However, this pattern differed between study sites. In Nashville, while those who agreed that "luck plays a big part in determining if I can keep from getting a sexually transmitted infection" reported a higher mean score 


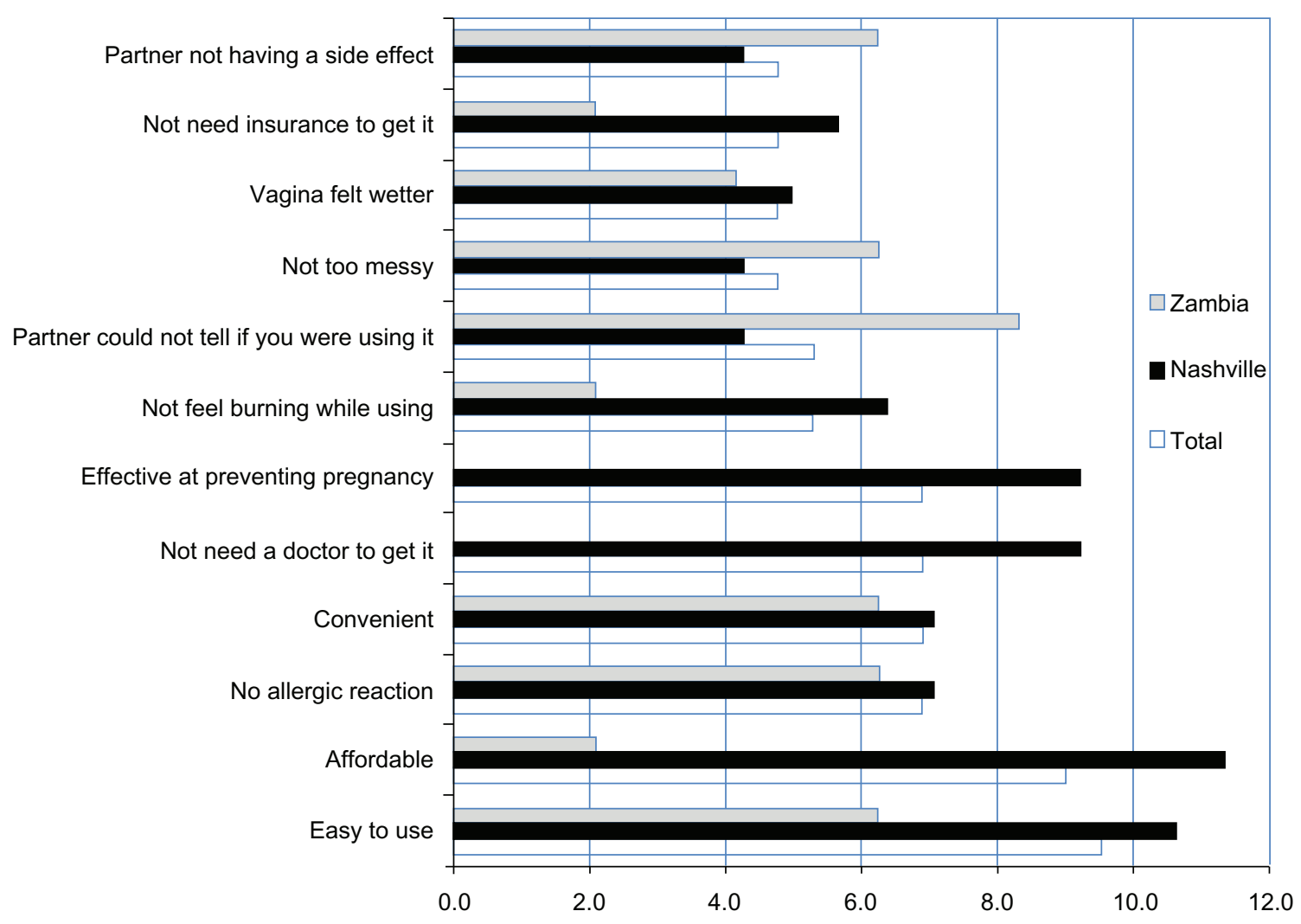

Figure I Proportion of top 12 reasons to like a vaginal microbicide cited by women enrolled in the study.

Notes: "Effective at preventing pregnancy," and "Not need a doctor to get it" were not cited as reasons by the Zambian cohort.

of conditional acceptability than those who disagreed with that statement. Those who agreed with "I feel almost certain that I will get AIDS" reported a lower mean score than those who disagreed. In Zambia, those who agreed with the following statements: "Regular consultation," "checks," and "wellness exams with a health care provider is the best way for me to avoid a sexually transmitted disease," "I refuse to have sex if my partner refuses to use a condom or latex barrier," "I feel almost certain that I will get AIDS," and "My past behavior puts me at high risk for getting HIV or AIDS" reported a significantly higher mean acceptability than those who disagreed with the same statements, respectively.

Among women in Zambia, 74\% of those who perceived they had a high risk for STIs responded that they would get HIV versus $20 \%$ of the women in Nashville. However, this comparison may not be statistically meaningful, as only 5 women in Nashville perceived themselves at high risk for STIs.

\section{Association of acceptability with sexual behaviors}

Willingness-to-use was marginally associated with condom use with a male partner in Nashville (65\% for never users of condoms and $48 \%$ for ever users; $P=0.076$ ) and use of barrier methods in Zambia (60\% for never users and 53\% for ever users; $P=0.090)$.

A series of ANOVA analyses determined that conditional acceptability was significantly associated with sexual behaviors. In Nashville, women who had one or more sex partners within the past 30 days and had never used a condom with a male partner were more likely to report a higher mean conditional acceptability than their counterparts, respectively. In Zambia, a significantly higher mean acceptability was found among those who ever used a barrier method and had never used a condom due to discomfort in asking a partner to use than their counterparts, respectively.

\section{Association of acceptability with STIs}

A higher proportion of willingness-to-use was found among those who had a history of one or more STIs (65\% for one or more STIs and $46 \%$ for non-STIs; $P=0.041$ ) only among the Nashville cohort. No significant association of STI history with conditional acceptability was reported in either site.

\section{Discussion}

One underexplored area of behavioral and social science research is an understanding of the social contexts in which a 
vaginal microbicide will be used. In this study, we endeavor to provide some information.

Although the majority of women at either site, Nashville (78.2\%) and Zambia (62.6\%), believed that their past behavior did not put them at risk for acquiring HIV or AIDS $(P<0.014)$; $48 \%$ of the Zambian cohort versus $4 \%$ of the Nashville cohort felt certain they would get AIDS $(P<0.0001)$. Among those in the Zambian cohort who felt they would acquire HIV, $87 \%$ indicated they were willing to use a vaginal microbicide product. In contrast, $>90 \%$ of women in both cohorts felt that if they took the right actions, they could avoid getting an STI. No difference was observed between the cohorts in the percentage of women indicating they could refuse to have sex if their partner "refuses to use a condom."

These findings suggest a high degree of acceptability toward use of a vaginal microbicide to prevent HIV infection. This study also demonstrated that women in the Zambian cohort view the risk of HIV infection as distinct from the risk of acquiring STIs and believe themselves to be limited in their ability to control whether they will be infected by HIV. This could be due to the fact that in Zambia, the two diseases are managed separately during treatment and are not particularly seen as STIs at the community level. In Zambia, the questionnaire used asked about HIV/AIDS and STI as separate questions and the outcomes for the Zambian cohort showed that the women viewed HIV infection as being completely different from acquiring an STI. HIV infection was not considered an STI but as a separate condition even though associated with their sexuality.

In Zambia, some of the women do not have full control of their health due to social demands on accepted behavior that a woman should demonstrate in a marriage setting. They may, in some cases, not have the capacity to negotiate for condom use with their partners, as this may be perceived to imply promiscuity. Most of the women reported they were married and many viewed themselves at risk of contracting HIV if their partners had other sexual partners. ${ }^{6}$ For those unable to negotiate mutual monogamy or condom use, the use of vaginal microbicide gels have been widely studied for decades with primarily limited success. Results of a study in sub-Saharan Africa with tenofovir gel, an antiretroviral microbicide, suggest its use could potentially fill an important HIV prevention gap. ${ }^{7}$ Karim et al found gel acceptability to be $97.4 \%$, with $97.9 \%$ indicating they would use tenofovir gel if it prevented HIV. However, they found that women with the highest gel adherence also tended to have the lowest reported coital frequency, with HIV incidence rates comparable (in the placebo arm) with those who reported much higher coital frequencies. ${ }^{7}$ The investigators noted this reflected both the study's location at the epicenter of southern Africa's HIV epidemic in the KwaZulu-Natal province and the very high-risk sex the women engaged in with migrant men. While ability of the coitally-related tenofovir gel to prevent HIV infection among this group was substantial, its decline in effectiveness after 18 months needs further investigation, including whether less gel was applied or adherence declined over time.

One recent study that investigated vaginal practices and associations with barrier methods and gel use among subSaharan African women enrolled in an HIV prevention trial found vaginal washing, wiping, and insertion of dry or absorbent material were all independently and inversely associated with consistent diaphragm and gel use with condom use. Van der Straten et $\mathrm{al}^{8}$ concluded that this knowledge, along with a better understanding of the sociocultural context in which these practices are embedded, could help improve education strategies to address potential modifiable behaviors.

Educational attainment is generally one of the key indicators for analyzing women's health status. Men and women tend to sustain a perpetual gender inequality within the family and society at large. Overall, the level of education in Zambia is higher among men than women and the proportion of women without education (ie, no schooling whatsoever) ranges from 4\% for women aged 15-20 years to $20 \%$ for women aged $45-49 .{ }^{6}$ Therefore, the women in the specific research setting in Zambia generally lack the capacity to demand their rights, which often has a negative impact on their health. In fact, being married is in itself a "risk factor," as they do not have a say in or control over the behavior of their partners. There are also high dropout rates among girls at primary and secondary levels, which may explain the reason why women believe themselves to be limited in their ability to control whether they will be infected by HIV or not, as marriage in the rural setting is seen as an economic gain. ${ }^{6}$

There were more women in Nashville than in Zambia who accepted the condition that the microbicide made the vagina wetter than normal during sex. This is due to the fact that in some African countries, the preference by men for a tight vagina, associated with virginity or fidelity, is emphasized via socialization. A study by Montgomery et $\mathrm{al}^{9}$ revealed that a number of male partners of women initiating gel use said that the added lubrication made it feel as though their partners had been sleeping with other men. For this reason, women said that if they did not tell their partners, their partners would not trust them and may think the women had been unfaithful. ${ }^{9}$ 


\section{Study limitation}

The study was conducted with few women in two rural sites and, as such, the results of this study cannot be generalized for Zambia. Another limitation in interpreting the results of the study is that data collection methods differed between countries (online survey for USA and personal interview for Zambia). Since online surveys provide respondents anonymity, results may reflect more honest answers to sensitive topics. However, a previous study ${ }^{10}$ has suggested that point estimation, but not patterns of associations, may be affected by the difference in survey methods. Another limitation is that because the study results resorted to quantitative data by asking close-ended questions, the full meaning of the results may not be clear, nor is cultural context entirely evident. This manuscript uses data based on the qualitative interviews to extrapolate the meaning of the results.

\section{Conclusion}

This study illustrates that women are willing to use the microbicide gel. However, there is a need to create awareness so that women can read about it, or have centers where they can go (preferably in a health care center or a community center) to access information on microbicide gels, especially in the Zambia site, where inadequate information was cited as a significant problem. Further, engaging men in the use of microbicide for a couple would contribute towards adherence so the women (mainly in the Zambian setting) would not live in fear of being accused of being unfaithful. The effectiveness of the gel was expressed as a concern for the women in Nashville and this could be addressed by ensuring that the pharmacologic properties are explained through literature.

\section{Acknowledgments}

We wish to acknowledge the survey participants. This study was supported in part from grants P20RR11792, G12RR003032, and U54RR022762. This paper is a result of the cooperation and collaboration between Nashville and
Zambia. In Nashville, we wish to acknowledge the support of the staff in the Center for Women's Health Research and the Center for AIDS Health Disparities Research at Meharry Medical College. In Zambia, sincere gratitude goes to the Provincial, District Directors, and members of staff from Kafue and Mumbwa districts hospitals, participants, and their staff for facilitating the undertaking of the study. We thank Debra Hughes for editorial support.

\section{Disclosure}

The authors report no conflicts of interest in this work.

\section{References}

1. McCormack S, Ramjee G, Kamali A, et al. PRO2000 vaginal gel for prevention of HIV-1 infection (Microbicides Development Programme 301): a phase 3, randomised, double-blind, parallel-group trial. Lancet. 2010;376(9749):1329-1337.

2. Buckheit RW, Watson KM, Morrow KM, Ham AS. Development of topical microbicides to prevent the sexual transmission of HIV. Antiviral Res. 2010;85(1):142-158.

3. El-Sadr WM, Mayer KH, Maslankowski L, et al. Safety and acceptability of cellulose sulfate as a vaginal microbicide in HIV-infected women. AIDS. 2006;20(8):1109-1116.

4. Ramjee G, Morar NS, Braunstein S, Friedland B, Jones H, van de Wijgert J. Acceptability of Carraguard, a candidate microbicide and methyl cellulose placebo vaginal gels among HIV-positive women and men in Durban, South Africa. AIDS Res Ther. 2007;4:20.

5. Whitehead SJ, Kilmarx PH, Blanchard K, et al. Acceptability of Carraguard vaginal gel use among Thai couples. AIDS. 2006;20(17):2141-2148.

6. Central Statistical Office (CSO), Ministry of Health (MOH), Tropical Disease Research Centre (TDRC), University of Zambia, and Macro International Inc. (2009). Zambia Demographic and Health Survey 2007. Calverton, Maryland, USA: CSO and Macro International Inc. Available from: http://www.measuredhs.com/pubs/pdf/FR211/ FR211\%5Brevised-05-12-2009\%5D.pdf. Accessed February 14, 2012.

7. Karim AQ, Karim SSA, Frohlich JA, et al; CAPRISA 004 Trial Group. Effectiveness and safety of tenofovir gel, an antiretroviral microbicide, for the prevention of HIV infection in women. Science. 2010;329(5996):1168-1174.

8. van der Straten A, Cheng H, Chidanykia A, De Bruyn G, Padian N. Vaginal practices and associations with barrier methods and gel use among Sub-Saharan African women enrolled in an HIV prevention trial. AIDS Behav. 2010;14(3):590-599.

9. Montgomery CM, Lees S, Stadler J, et al. The role of partnership dynamics in determining the acceptability of condoms and microbicides. AIDS Care. 2008;20(6):733-740.

10. Heiervang E, Goodman R. Advantages and limitations of web-based surveys: evidence from a child mental health survey. Social Psychiatry Psychiatr Epidemiol. 2011;46(1):69-76.
HIV/AIDS - Research and Palliative Care

\section{Publish your work in this journal}

HIV/AIDS - Research and Palliative Care is an international, peerreviewed open-access journal focusing on advances in research in HIV, its clinical progression and management options including antiviral treatment, palliative care and public healthcare policies to control viral spread. The journal welcomes original research, basic science,

\section{Dovepress}

clinical \& epidemiological studies, reviews \& evaluations, expert opinion \& commentary, case reports \& extended reports. The manuscript management system is completely online and includes a very quick and fair peer-review system. Visit http://www.dovepress.com/ testimonials.php to read real quotes from published authors. 